\title{
Non-Invasive Magnetocardiography for the Early Diagnosis of Coronary Artery Disease in Patients Presenting With Acute Chest Pain
}

\author{
Hyukchan Kwon, PhD; Kiwoong Kim, PhD; Yong-Ho Lee, PhD; Jin-Mok Kim, PhD; \\ Kwon Kyu Yu, BSc; Namsik Chung, MD, PhD; Young-Guk Ko, MD, PhD
}

\begin{abstract}
Background: Accurate identification of patients with acute coronary syndrome (ACS) is often difficult especially when an electrocardiogram (ECG) does not show typical elevation of ST segment. The aim of the present study was therefore to evaluate the efficacy of magnetocardiography (MCG) for diagnosis of ACS in patients with acute chest pain presenting without ST segment elevation.

Methods and Results: In the present retrospective study 364 patients with the suspected ACS without ST segment elevation were selected. Significant coronary artery disease (CAD) was defined as a stenosis $\geq 50 \%$ in at least one of 16 segments of the 3 major coronary arteries and their branches. The MCG recordings were obtained at resting state using a 64-channel MCG system in a magnetically shielded room. The patients were classified on the basis of the probability distribution. The presence of significant CAD was identified with a sensitivity of $84.0 \%$ and a specificity of $85.0 \%$, compared to $44.7 \%$ and $89.8 \%$ on ECG. In the subgroup of patients without specific findings on ECG or biomarker test, MCG had a sensitivity of $73.5 \%$ and a specificity of $82.3 \%$.
\end{abstract}

Conclusions: MCG was acceptably sensitive and specific in identifying patients with ACS even in the absence of specific findings on ECG and positive biomarker tests. Thus, MCG seems beneficial for the early triage of patients with acute chest pain. (Circ J 2010; 74: 1424-1430)

Key Words: Acute coronary syndrome; Coronary artery disease; Magnetocardiography; Non-invasive detection

$\mathbf{P}$ atients presenting with acute chest pain without ST elevation on electrocardiogram are a diverse and heterogeneous group representing a wide spectrum of disease severity from acute myocardial infarction (MI) to non-ischemic chest pain syndromes. Despite development of various diagnostic approaches and modern treatments, the rates of mortality, MI and readmission in acute coronary syndrome (ACS) remain very high. ${ }^{1}$ In general, initial risk assessment in patients with acute chest pain is performed on the basis of the clinical, electrocardiographical and biochemical data to identify patients with acute myocardial ischemia. ${ }^{2}$ In high-risk patients, coronary angiography is required to confirm the clinical diagnosis, and subsequent coronary revascularization either on percutaneous coronary intervention or coronary artery bypass graft is considered, depending on the severity of coronary artery disease (CAD). Because of inherent risks and relatively high costs, however, the use of coronary angiography is restricted to patients judged to be at high risk for myocardial progression to MI or death. ${ }^{3}$ Thus, if magnetocardiography (MCG) can effectively identify patients with myocardial ischemia before coronary angiography, it will be a valuable non-invasive technique for risk stratification at an early stage of admission.

MCG has been proposed as a non-invasive and contactfree technique for functional diagnosis of the heart such as cardiac ischemia and arrhythmia. ${ }^{4-8}$ MCG records the magnetic field induced by the same bioelectric currents recorded on electrocardiography (ECG). ${ }^{4}$ During myocardial ischemia, ST segment and T wave changes often appear on ECG. Acute ischemia usually results in transient horizontal or downsloping ST segment depression and T wave flattening or inversions in ECG.9.10 Because these changes originate from the abnormal current flow in the myocardium during ventricular repolarization, magnetic field orientation correlated with the current flow was used for ischemia decision in resting ${ }^{11-16}$ and stress-testing MCG. ${ }^{17,18}$ In principle, MCG is more sensi-

Received December 7, 2009; revised manuscript received March 11, 2010; accepted March 12, 2010; released online May 27, 2010 Time for primary review: 30 days

Center for Brain and Cognitive Science Research (H.K., K.K., Y.-H.L., J.-M.K., K.K.Y.), Korea Research Institute of Standards and Science, Daejeon; Cardiology Division (N.C., Y.-G.K.), Department of Internal Medicine, Yonsei University Medical College, Seoul, Korea

Mailing address: Namsik Chung, MD, PhD, Cardiology Division, Department of Internal Medicine, Yonsei University Medical College, Seoul, Korea. E-mail: namsikc@yuhs.ac

ISSN-1346-9843 doi:10.1253/circj.CJ-09-0975

All rights are reserved to the Japanese Circulation Society. For permissions, please e-mail: cj@j-circ.or.jp 


\begin{tabular}{|lccc|}
\hline \multicolumn{4}{|c|}{ Table 1. Patient Characteristics } \\
& $\begin{array}{c}\text { CAD } \\
(\mathbf{n = 2 3 7})\end{array}$ & $\begin{array}{c}\text { Minimal CAD } \\
(\mathbf{n = 1 2 7})\end{array}$ & $\begin{array}{c}\text { Normal } \\
(\mathbf{n}=\mathbf{8 9})\end{array}$ \\
Female, $\mathrm{n}(\%)$ & $80(34)$ & $74(58)$ & $42(47)$ \\
Age (years) & $61.3 \pm 10.3$ & $55.0 \pm 10.9$ & $24.5 \pm 5.2$ \\
Coronary status, $\mathrm{n}(\%)$ & & & \\
1-vessel disease & $89(38)$ & 0 & - \\
2-vessel disease & $79(33)$ & 0 & - \\
3-vessel disease & $69(29)$ & 0 & - \\
Abnormal ECG, $\mathrm{n}(\%)$ & $106(45)$ & $13(10)$ & 0 \\
RWMA, $\mathrm{n}(\%)$ & $99(42)$ & $2(2)$ & - \\
Disease & & & \\
$\quad$ Atypical chest pain, $\mathrm{n}(\%)$ & $18(8)$ & $99(78)$ & - \\
Unstable angina, $\mathrm{n}(\%)$ & $128(54)$ & $28(22)$ & - \\
$\quad$ Ml, $\mathrm{n}(\%)$ & $91(38)$ & 0 & - \\
Ejection fraction, (\%) & $59.4 \pm 12.4$ & $67.7 \pm 7.4$ & - \\
Diabetes mellitus, $\mathrm{n}(\%)$ & $49(21)$ & $7(6)$ & 0 \\
Hypertension, $\mathrm{n}(\%)$ & $75(32)$ & $36(27)$ & 0 \\
\hline
\end{tabular}

CAD, coronary artery disease; ECG, electrocardiography; RWMA, regional wall motion abnormality; $\mathrm{MI}$, myocardial infarction.

tive to tangential currents than ECG. MCG is also sensitive to circular vortex currents not detectable on ECG, and it has been shown to contain information complementary to ECG. Thus MCG may show pathological deviations from the normal activation direction induced by, for example, myocardial ischemia, with better accuracy than ECG.

For CAD detection in the resting state, several studies have reported that MCG is superior to ECG in that it has a diagnostic accuracy of $60-90 \%$ in various patient populations. ${ }^{11-16}$ Comparing spatial distribution of QT dispersion, a sensitivity of $74 \%$ and a specificity of $80 \%$ were reported between 23 CAD patients and a control group, in which 13 out of 20 subjects in the control group were normal subjects. ${ }^{11}$ In comparison between patients with and without hemodynamic relevant stenosis, a sensitivity of $62.8 \%$ and a specificity of $61.3 \%$ were reported on analysis of the current density vector. ${ }^{12}$ A sensitivity and a specificity of $>90 \%$ were recently reported in patients admitted to the intensive care unit, using four variables derived during ST-T interval. ${ }^{13}$ But in that study 63 out of 264 datasets had to be excluded due to insufficient signal-to-noise ratio, because they measured MCG with a 9-channel system in an unshielded environment. In contrast, another group reported a sensitivity of $76.4 \%$ and a specificity of $74.3 \%$ in a similar situation. ${ }^{14}$ Although these results demonstrated the usefulness of MCG, there are some limitations in diagnostic accuracy and patient population. For timely and accurate diagnosis of CAD, therefore, there is a need for a better classification method of MCG parameters depending on the severity of ischemia to improve diagnostic accuracy.

In a preliminary study we proposed a new classification method for detection of CAD on the basis of different distribution of the MCG parameters between CAD patients and symptomatic patients or healthy volunteers. ${ }^{19}$ The aim of the present study was to validate the method in a relatively large population as a useful tool for the early diagnosis of CAD (stenosis $\geq 50 \%$ ) in patients with ACS without ST elevation.

\begin{tabular}{|ll|}
\hline $\begin{array}{l}\text { Table 2. MCG Parameters } \\
\text { Abbreviation }\end{array}$ & \multicolumn{1}{c|}{ Definition } \\
TT_CAMx & $\begin{array}{l}\text { Maximum value of the main current angle in the } \\
\text { ST period. }\end{array}$ \\
TT_CMD & $\begin{array}{l}\text { Maximum value of a dynamic change of the } \\
\text { strength of the main current vector within a time } \\
\text { interval of } 30 \mathrm{~ms} \text { in the ST period. }\end{array}$ \\
& $\begin{array}{l}\text { Orientation of magnetic field map at Tmax. } \\
\text { T_FMA }\end{array}$ \\
R_FMA & Orientation of magnetic field map at Rpeak.
\end{tabular}

MCG, magnetocardiography; TT_, ST period; T_, peak of T-wave; $R_{-}$, peak of R-wave.

\section{Methods}

\section{Subjects}

The present study was performed with the approval of the institutional review board at Yonsei University Medical College. Written informed consent was obtained from all subjects. For this retrospective study, 364 consecutive patients, who were admitted to the cardiovascular center with suspected ACS between May 2004 and April 2006, were enrolled. Exclusion criteria were persistent ST elevation, suspected variant angina, any specific abnormalities of bundle branch block, atrial fibrillation, arrhythmia on ECG or left ventricular hypertrophy on echocardiography. Known CAD patients with old MI or previous revascularizations, and MI patients with insignificant coronary stenosis $(<50 \%)$ were also excluded. Out of 364 patients, 329 patients underwent coronary angiography during the initial hospitalization. Abnormal ECG changes indicative of myocardial ischemia without persistent ST elevation such as ST depression, T inversion and pathologic Q waves were observed in 119 patients. On echocardiogram, regional wall motion abnormality was observed in 101 out of 317 patients. On biomarker tests, 91 patients had elevated troponin $\mathrm{T}$ level $(>0.1 \mathrm{ng} / \mathrm{ml})$ level or peak creatine kinase $>2$ times the upper limit of normal, indicating MI. Unstable angina (UA) was diagnosed in 156 patients. UA was defined as angina pectoris or an equivalent type of ischemic discomfort with $\geq 1$ of the following 3 features and negative results of cardiac biomarker tests (such as troponin T and creatine kinase-MB): (1) prolonged (usually $\geq 20$ min) angina occurring at rest; (2) newonset (within 1 month) angina of Canadian Cardiovascular Society class $\geq$ III severity; and (3) previously diagnosed angina that had become distinctly more frequent, longer in duration, or lower in threshold.

Based on the severity of CAD, patients were divided into 2 groups: those with stenosis $\geq 50 \%$ in at least 1 of 16 segments in the 3 major coronary arteries and their branches, termed the CAD group ( $n=237$, mean age $61.3 \pm 10.3$ years); and those with stenosis $<50 \%$ at all 16 segments or who were discharged without undergoing coronary angiography, termed the minimal CAD group $(n=127$, mean age 55.0 \pm 10.9 years $)$. In the CAD group 89 patients had single-vessel disease, 79 had 2-vessel disease, and the remaining 69 patients had 3vessel disease. As a reference, the normal group (mean age $24.5 \pm 5.2$ years) consisted of 89 healthy volunteers with no history of any cardiovascular disease and with normal ECG at rest. The clinical characteristics of all subjects are listed in Table 1. Some of the MCG data have been used in previous studies. ${ }^{15-17,19}$

\section{MCG Recording}

In patients who underwent coronary angiography, MCG re- 


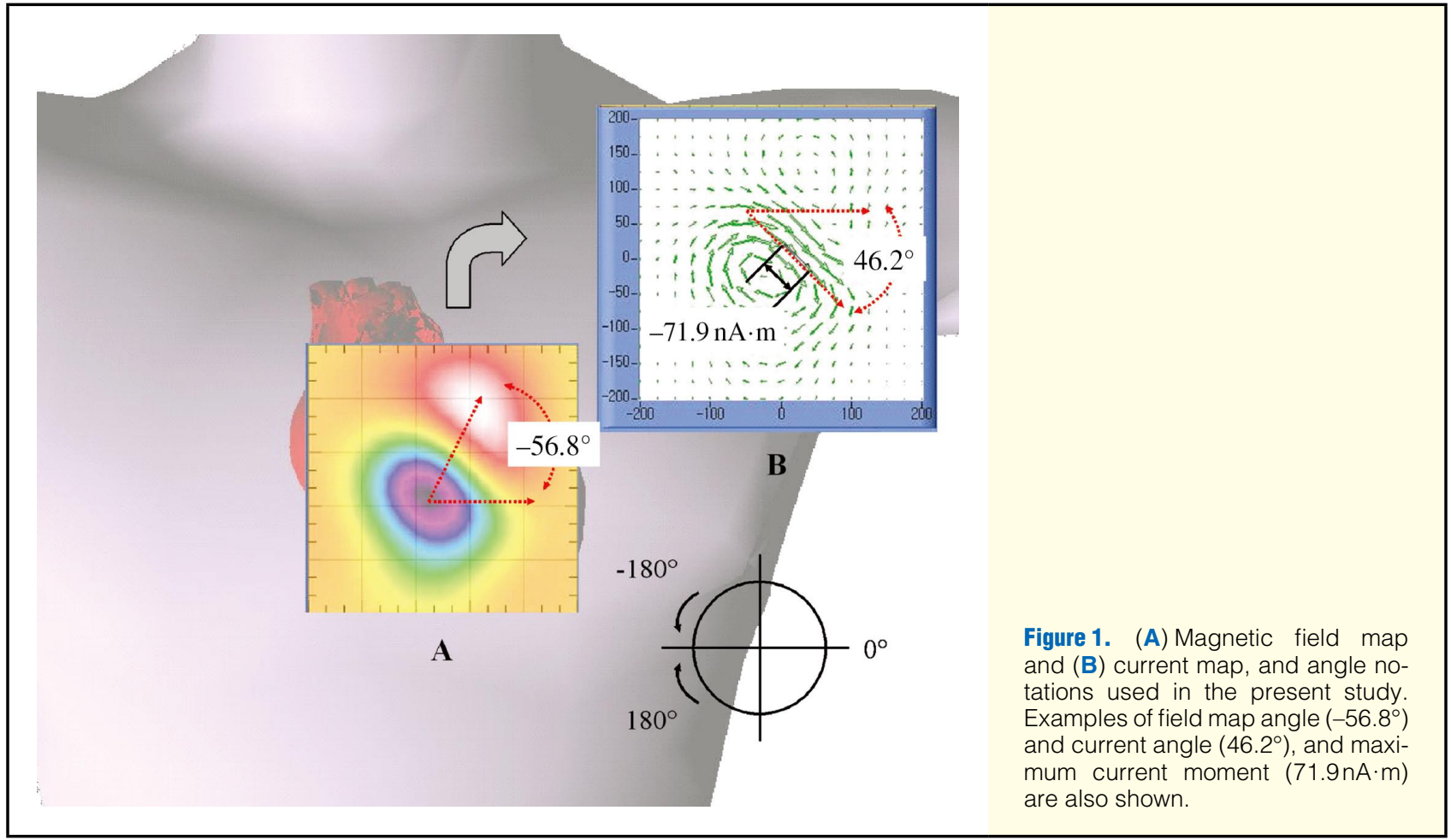

A

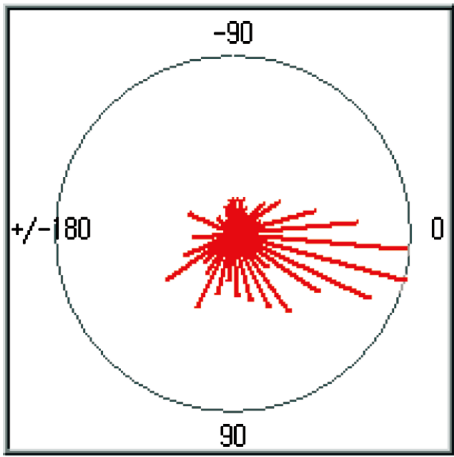

CAD group
B

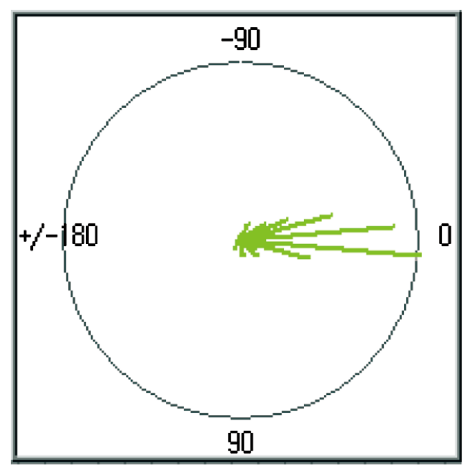

minimal CAD group
C

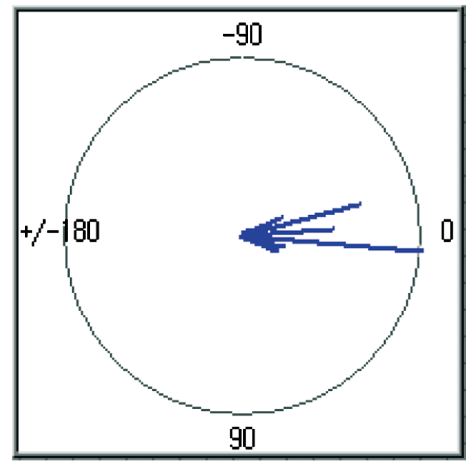

normal group

Figure 2. T_FMA-R_FMA (orientation of magnetic field map at Tmax relative to the orientation of magnetic field map at Rpeak) in the (A) CAD, (B) minimal CAD and (C) normal groups, respectively. CAD, coronary artery disease.

cordings were obtained prior to coronary angiography. All patients were treated and stabilized according to the clinical presentation. All patients were chest pain free at the time of MCG recording. MCG was recorded in the resting state using a multichannel MCG system inside a magnetically shielded room. ${ }^{20} \mathrm{MCG}$ measures cardiac magnetic fields tangential to the chest surface at 64 locations using 1st-order planar gradiometers in an area of $162 \mathrm{~mm} \times 162 \mathrm{~mm}$ with a sensor interval of approximately $35 \mathrm{~mm}$. The total recording time was $<10 \mathrm{~min}$. The MCG signals were digitally recorded for $30 \mathrm{~s}$ at the sampling rate of $500 \mathrm{~Hz}$. After baseline correction, ${ }^{21}$ data were averaged using R-peaks. The averaged signals were analyzed after DC offset correction using a baseline during the $\mathrm{P}-\mathrm{Q}$ interval.

\section{MCG Parameters}

Three MCG parameters were derived from the spatial distribution of the measured magnetic fields during the repolarization period (Table 2). Magnetic field map (MFM) angle at $T_{\max }, T_{-}$FMA, was measured in respect to the patient's right-left line as shown in Figure 1. After the source current map was reconstructed from the spatial distribution of the cardiac fields using a distributed current source model and minimum norm estimation algorithm, ${ }^{22}$ angle and strength of the main current vector were calculated at each time instant during the ST period between the J-point and $\mathrm{T}_{\max }$. Among 


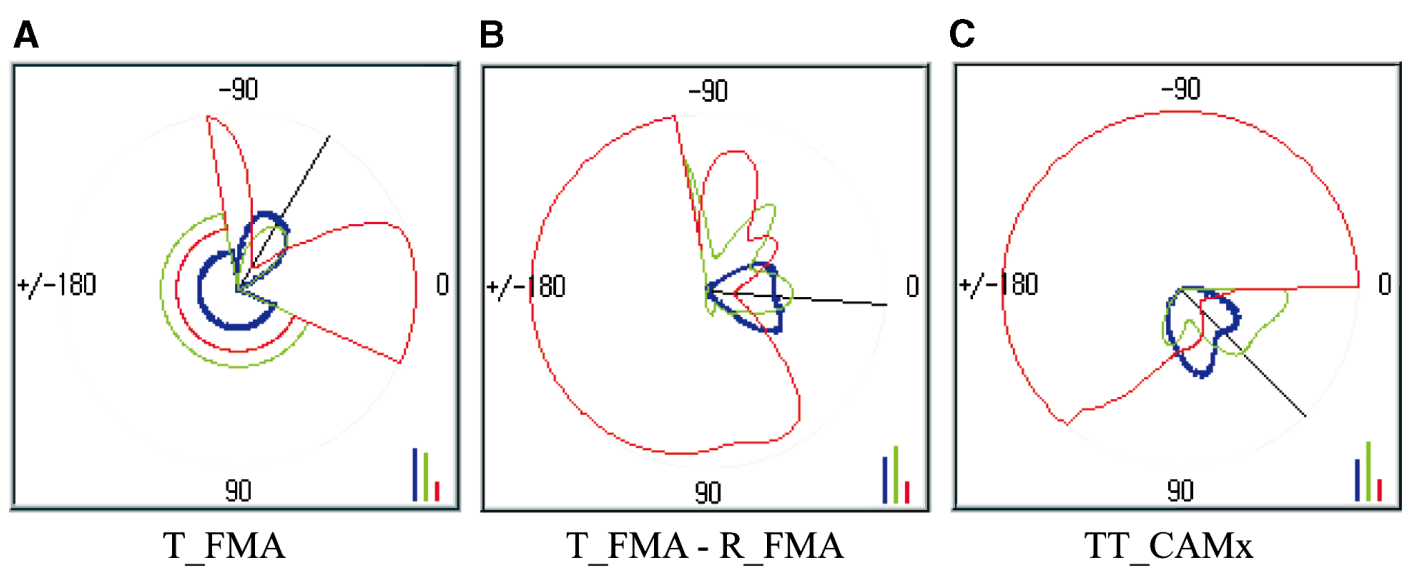

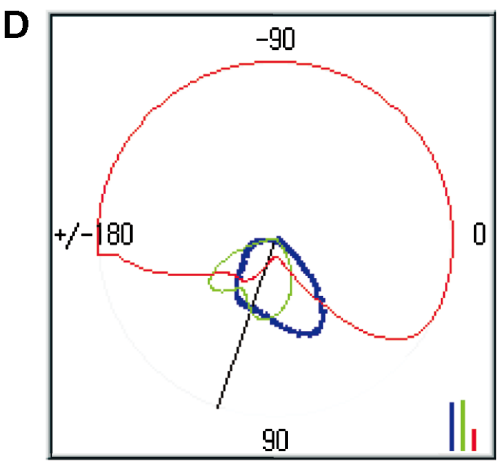

TT_CAMx - R_FMA

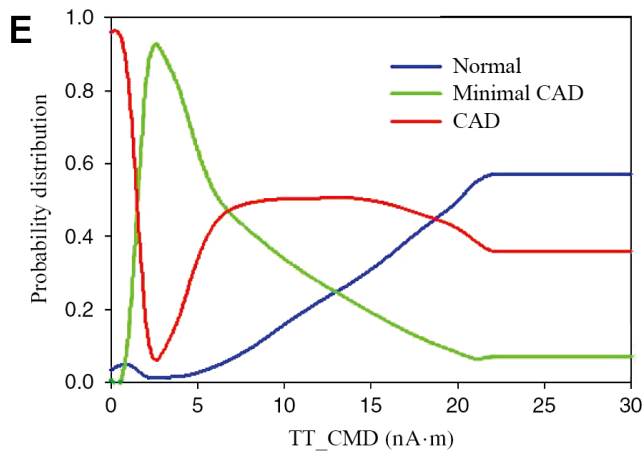

TT_CMD

Figure 3. Conditional probability $P\left(g_{i} \mid x_{j}\right)$ that a subject belongs to the group $g_{i}$ (normal, thick; minimal CAD, gray; CAD, thin) given that he/she has the measured value $\boldsymbol{x}_{\mathrm{j}}$ for the variables (A) T_FMA (orientation of magnetic field map at Tmax), (B) T_FMAR_FMA (orientation of magnetic field map at Tmax relative to the orientation of magnetic field map at Rpeak), (C) TT_CAMx (maximum of the main current angle in the ST period), (D) TT_CAMx-R_FMA and (E) TT_CMD (maximum of a dynamic change of the strength of the main current vector within a time interval of $30 \mathrm{~ms}$ in the ST period), respectively. Straight radius line, measured variable. For example, when $\boldsymbol{x}_{1}\left(\mathrm{~T}_{-} \mathrm{FMA}\right)=-56.8^{\circ}$ in $(\mathrm{a}), P\left(g_{i} \mid \boldsymbol{x}_{1}\right)=0.448,0.409$ and 0.144 in the normal, minimal CAD and CAD groups, respectively, as plotted in the bar chart in the lower right corners. CAD, coronary artery disease.

them, the maximum of the current angle was TT_CAMx and its dynamic change within a 30-ms interval was TT_CMD. The early time window of the ST period, however, when the main current vector could not be definitely evaluated from the MFM due to the small signals or circulating current pattern, was excluded from the analysis. Additionally, MFM angle at $R_{\text {peak }}$ was used as a reference angle to reduce the effect of different electrical axis between individuals. ${ }^{23}$ Five variables were therefore used for diagnosis: T_FMA, T_FMAR_FMA, TT_CAMx, TT_CAMx-R_FMA and TT_CMD.

\section{Algorithm of Weighted Maximum a Posteriori (wMAP)}

In the wMAP method, the probability that a patient has $\mathrm{CAD}$ is represented as a function of 5 variables. The probability was calculated from the prior knowledge on the probability distribution, which was estimated from MCG data collected in 2004 (86 CAD patients, mean age 60.3 \pm 10.1 years; 59 minimal CAD patients, mean age $52.9 \pm 10.2$ years; 66 healthy volunteers, mean age $22.8 \pm 3.3$ years). As an example, Figure 2 shows the histogram of T_FMA-R_FMA (the orientation of MFM at $T_{\max }$ relative to the orientation of MFM at $\mathrm{R}_{\text {peak }}$ ) in the CAD, minimal CAD and normal group, respectively.
Interpolating the histograms and normalizing by the total number of subjects in each group, a probability distribution function was estimated and it was defined as the conditional probability $P\left(x_{j} \mid g_{i}\right)$ that a subject has the value $x_{j}$ given that the subject belongs to the group $g_{i}$. Here $g_{i}$ are indexing the groups of normal, minimal CAD and CAD, respectively, and $x_{j}$ is one of the 5 variables, T_FMA, T_FMA-R_FMA, TT_ CAMx, TT_CAMx-R_FMA and TT_CMD. Accordingly, the posterior probability $P\left(g_{i} \mid x_{j}\right)$ that a subject belongs to the group $g_{i}$ given that he/she has the measured value $x_{j}$, can be estimated by the Bayes theorem as follows:

$$
P\left(g_{i} \mid x_{j}\right)=\frac{P\left(x_{j} \mid g_{i}\right) \cdot P\left(g_{i}\right)}{\sum_{k} P\left(x_{j} \mid g_{k}\right) \cdot P\left(g_{k}\right)}
$$

where $P\left(g_{i}\right)$ is the prior probability of a subject to belong to the group $g_{i}$ and is assumed to be the same for all groups. Figure 3 shows the estimated posterior probability $P\left(g_{i} \mid x_{j}\right)$ for the 5 variables.

Next, the probability $P\left(g_{i} \mid x_{1}, x_{2}, x_{3}, x_{4}, x_{5}\right)$ that a subject belongs to the normal, minimal CAD, or CAD group is calculated as a weighted sum of the posterior probabilities by Eq. (1) in each group as follows: 


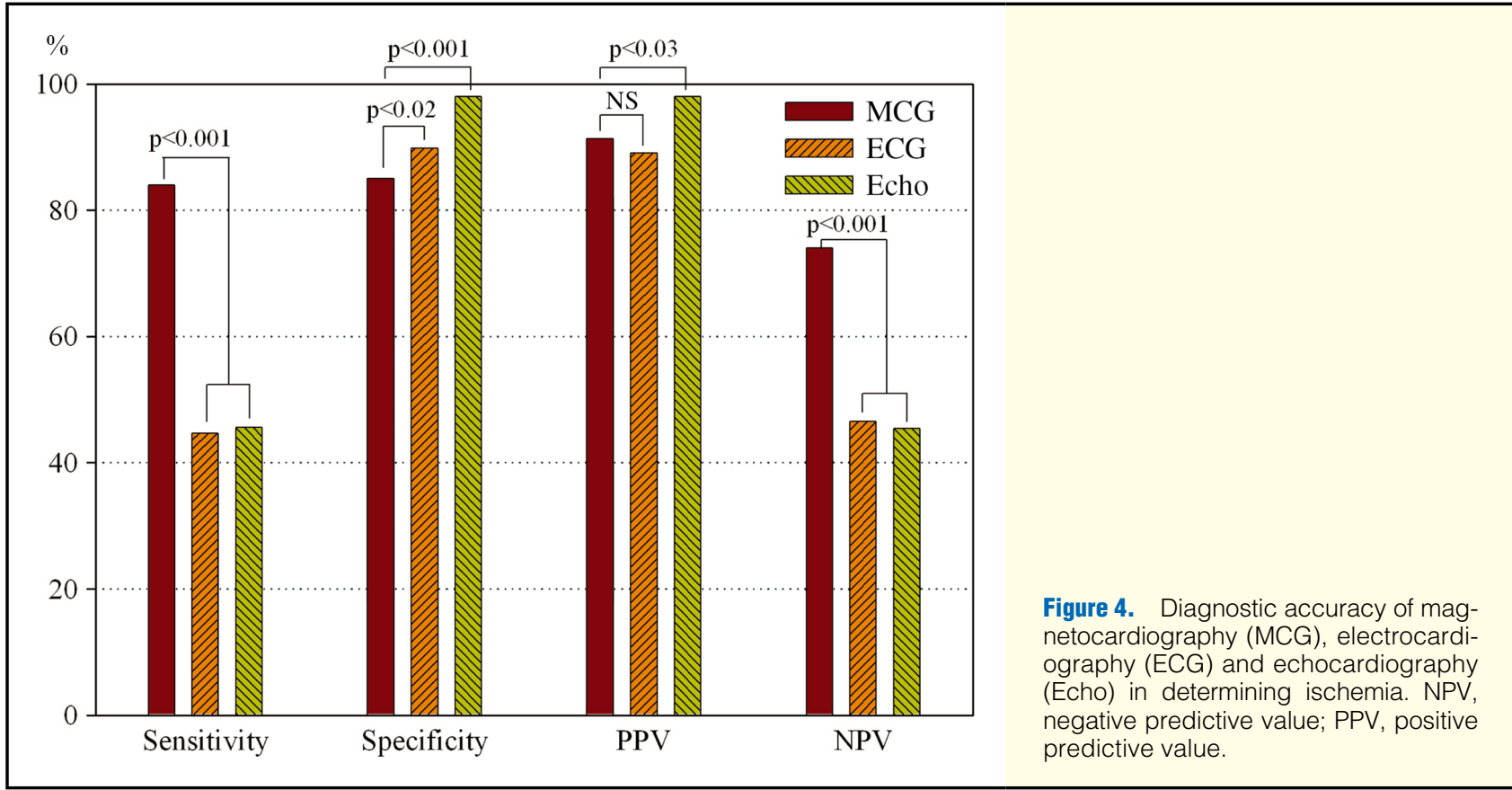

\begin{tabular}{|c|c|c|c|c|c|c|c|}
\hline \multirow{2}{*}{ Including criteria } & \multicolumn{2}{|c|}{ No. patients } & \multirow{2}{*}{ Tool } & \multirow{2}{*}{$\begin{array}{c}\text { Sensitivity } \\
(\%)\end{array}$} & \multirow{2}{*}{$\begin{array}{c}\text { Specificity } \\
(\%)\end{array}$} & \multirow{2}{*}{$\begin{array}{l}\text { PPV } \\
\text { (\%) }\end{array}$} & \multirow{2}{*}{$\begin{array}{l}\text { NPV } \\
(\%)\end{array}$} \\
\hline & CAD & Minimal CAD & & & & & \\
\hline \multirow[t]{3}{*}{ All patients } & & & MCG & 84.0 & 85.0 & 91.3 & 74.0 \\
\hline & 237 & 127 & ECG & 44.7 & 89.8 & 89.1 & 46.5 \\
\hline & & & & $P<0.001$ & $P<0.02$ & NS & $P<0.001$ \\
\hline Negative biomarker & & & MCG & 78.1 & 82.6 & 87.7 & 70.4 \\
\hline \multirow[t]{2}{*}{ Angiogram available } & 146 & 92 & ECG & 30.1 & 85.9 & 77.2 & 43.6 \\
\hline & & & & $P<0.001$ & NS & NS & $P<0.001$ \\
\hline \multicolumn{8}{|l|}{ Negative biomarker } \\
\hline No specific findings on ECG & 102 & 79 & MCG & 73.5 & 82.3 & 84.3 & 70.7 \\
\hline Angiogram available & & & & & & & \\
\hline
\end{tabular}

$P$ was calculated for the comparison of diagnostic accuracy between MCG and ECG.

PPV, positive predictive value; NPV, negative predictive value. Other abbreviations see in Tables 1,2.

$$
P\left(g_{i} \mid x_{1}, x_{2}, x_{3}, x_{4}, x_{5}\right)=\sum_{j} w_{j} P\left(g_{i} \mid x_{j}\right)
$$

Finally, the presence of significant CAD is determined by the weighted sum of the posterior probability of belonging to CAD in comparison to the probability of belonging to the minimal CAD or normal groups. A subject is classified as having significant $\mathrm{CAD}$ if $\mathrm{P}\left(\mathrm{CAD} \mid x_{1}, x_{2}, x_{3}, x_{4}, x_{5}\right)$ is larger than $\mathrm{P}\left(\right.$ minimal $\left.\mathrm{CAD} \mid x_{1}, x_{2}, x_{3}, x_{4}, x_{5}\right)$ and $\mathrm{P}$ (normal $\mid x_{1}, x_{2}$, $\left.x_{3}, x_{4}, x_{5}\right)$. Otherwise, he/she is classified as not having significant CAD. The weighting factors were adjusted experimentally so that the diagnostic accuracy is maximized. The best classification result was obtained with the weighting factors of $0.3,1.0,2.0,1.7$ and 2.5 for T_FMA, T_FMAR_FMA, TT_CAMx, TT_CAMx-R_FMA and TT_CMD, respectively.

\section{Statistical Analysis}

Data are reported as mean \pm SD for continuous variables, or as percentages for categorical variables. For the angular parameter, the mean was calculated as the inverse tangent of the mean of the cosines and sines of each angle. In order to estimate a probability distribution function, the histogram was represented by a combination of normal distribution function and gamma distribution function. Sensitivity, specificity, positive predictive value (PPV) and negative predictive value (NPV) were determined in comparison between the CAD group and minimal CAD group according to the contingency table. Differences in categorical data were assessed on chisquare test. Statistical significance was defined as $\mathrm{P}<0.05$.

\section{Results}

All patients were classified according to the wMAP method using the prior probability distribution (Figure 3) and weighting factors. As a result, 199 out of 237 patients in the CAD group were classified as having CAD on MCG, and 108 out of 127 patients in minimal CAD group had negative MCG signs, giving a sensitivity, specificity, PPV and NPV of $84.0 \%$, $85.0 \%, 91.3 \%$ and $74.0 \%$, respectively $(\mathrm{P}<0.001)$. In the normal group, 87 out of 89 subjects had negative MCG signs $(97.8 \%)$. The diagnostic accuracy of MCG was compared with 
that of the 12-lead ECG and echocardiography in Figure 4, showing a significantly higher sensitivity and NPV for MCG than ECG and echocardiography $(\mathrm{P}<0.001)$. In contrast, the specificity of MCG was lower than for ECG $(\mathrm{P}<0.02)$ or echocardiography $(\mathrm{P}<0.001)$, and the PPV of MCG was lower than that of echocardiography $(\mathrm{P}<0.03)$, but the differences were not so large. Here an ECG was defined as positive in the case of pathologic Q wave, amplitude of ST depression $\geq 0.05 \mathrm{mV}$ or $\mathrm{T}$ inversion $\geq 0.2 \mathrm{mV} .{ }^{24}$ The diagnostic accuracy of MCG was also tested in a conditioned population. In the subgroup of 238 patients who had no indications of ischemia on biochemistry but who underwent coronary angiography due to suspected CAD, MCG still had a higher sensitivity of $78.1 \%$ and NPV of $70.4 \%(\mathrm{P}<0.001)$, compared to that of $30.1 \%$ and $43.6 \%$, respectively, on ECG. But the specificity and PPV of MCG were similar to those of ECG $(\mathrm{P}>0.05)$. When 57 patients with indications of ischemia on ECG were further excluded from the subgroup, MCG had a sensitivity of $73.5 \%$ and NPV of $70.7 \%$ in the remaining 181 patients $(\mathrm{P}<0.001)$. The results are summarized in Table 3 .

\section{Discussion}

The present retrospective study analyzed MCG datasets of 364 patients with suspected ACS without ST elevation and was focused on the diagnostic accuracy in the heterogeneous population for practical use in a general clinical environment. Diagnostic accuracy was then determined in comparison between patients with and without severe CAD. Furthermore, severe $\mathrm{CAD}$ was defined as stenosis $\geq 50 \%$ at not only major coronary arteries but also their branches for the detection of CAD in an early stage of stenosis. Therefore, 23 patients, who would have been diagnosed as having minimal CAD on general criteria of a stenosis $\geq 70 \%$ in a major coronary artery, were additionally included in the CAD group. Among them, 18 patients were classified as having CAD on MCG (78.3\%).

It is well known that when there is ischemia, biological injury currents are generated that can be detected on MCG. Therefore an abnormality in cardiac depolarization or repolarization is reflected in an abnormality in the MFM. ${ }^{5}$ But a diagnostic method for CAD detection has not been established. Here, we used an abnormality of several MCG parameters, such as the orientation angle derived from MFM and the current vector, and a dynamic change in the strength of the main current vector during the time interval between the beginning of the $\mathrm{T}$ wave and $\mathrm{T}$ wave apex. In general, abnormality of MCG parameters was determined dichotomously so that the probability of having CAD is 1 or 0 depending on whether the parameter exceeds the normal range or not. Such binary decision might be useful in discriminating between a high-risk group (UA or MI) and a healthy group. ${ }^{16}$ As shown in Figure 2, however, it is limited in the heterogeneous population because a number of CAD patients have MCG parameters in the intermediate region between normal and abnormal ranges. Using our method, the presence of CAD is determined by comparing the probability, which was calculated from the prior knowledge on the probability distribution of the MCG parameters depending on the presence of CAD. In this way we obtained better diagnostic accuracy than the previous studies, ${ }^{11-14}$ showing that the proposed method is more effective than the conventional binary decision method. This wMAP method was tested with a small population (86 CAD patients, 59 minimal CAD patients, 66 healthy volunteers) and was applied to all patients to confirm the validity in a relatively large population. The results demonstrate that the diagnostic criteria proposed in the present study may not lose the ability to correctly classify newly added patients.

Compared with ECG and echocardiography, MCG had distinctly higher sensitivity and NPV (Figure 4). The results show that MCG may be useful to avoid the inadvertent discharge of the patient who truly has myocardial ischemia. The difference of diagnostic accuracy between MCG and ECG was further tested in a conditioned population (Table 3 ). First, 91 patients, who clearly had high probability of CAD due to positive biomarkers, and 35 patients, who were discharged without undergoing coronary angiography due to high probability of minimal CAD, were excluded. In this subgroup of 238 patients, MCG still had higher sensitivity and NPV with similar specificity and PPV, compared to ECG. When 57 patients with indications of ischemia in ECG were further excluded from the subgroup, MCG had a sensitivity of $73.5 \%$ and NPV of $70.7 \%$ in the remaining 181 patients. These results demonstrate the efficacy of MCG as a complementary tool to ECG in patients with no indication of ischemia according to various diagnostic tools before angiography.

\section{Study Limitations}

First, MCG measures abnormal electrophysiological changes caused by ischemia, and the severity of ischemia was known to be affected by the degree and morphology of stenosis. ${ }^{25}$ In the present study the severity of CAD was defined by the degree of stenosis on coronary angiography and we need a clear gold standard for ischemia. Second, our classification method is based on the probability distribution function, examined in a retrospective study and the findings have not been prospectively validated. For clinical application of the method, the diagnostic accuracy should be accepted reliably without loss of the classification accuracy when new cases are added in the future. Finally, the presence of CAD is represented as a probability without electrophysiological or anatomical information. Further work to localize the ischemic region in a realistic heart model is needed.

In conclusion, MCG was acceptably sensitive and specific in identifying patients with ACS even in the absence of specific findings on ECG and positive biomarker tests. Thus, MCG seems beneficial for the early triage of patients with acute chest pain.

\section{References}

1. Battler A. European heart survey of acute coronary syndromes. Eur Heart J 2002; 23: 1190-1201.

2. Bertrand ME, Simoons ML, Fox KAA, Wallentin LC, Hamm CW, McFadden E, et al. Management of acute coronary syndromes in patients presenting without persistent ST-segment elevation. Eur Heart J 2002; 23: 1809-1840.

3. Scanlon PJ, Faxon DP, Audet AM, Carabello B, Dehmer GJ, Eagle $\mathrm{KA}$, et al. ACC/AHA guidelines for coronary angiography: A report of the American College of Cardiology/American Heart Association Task Force on Practice Guidelines (Committee on Coronary Angiography) developed in collaboration with the Society for Cardiac Angiography and Interventions. J Am Coll Cardiol 1999; 33: $1756-1824$.

4. Malmivuo J, Plonsey R. Bioelectromagnetism: Principles and applications of bioelectric and biomagnetic fields. New York: Oxford University Press, 1995.

5. Stroink G, Moshage W, Achenbach S. Cardiomagnetism. In: Andrä W, Nowak H, editors. Magnetism in Medicine. Wiley-VCH, Berlin: 1998; 136-189.

6. Tavarozzi I, Comani S, Del Gratta C, Di Luzio S, Romani GL, Gallina S, et al. Magnetocardiography: Current status and perspectives. Part II: Clinical applications. Ital Heart J 2002; 3: 151-165.

7. Fenici R, Brisinda D, Meloni AM. Clinical application of magnetocardiography. Expert Rev Mol Diagn 2005; 5: 291-313. 
8. Joung B, Kim K, Lee YH, Kwon H, Lim HK, Kim TU, et al. Magnetic dispersion of the late repolarization in Brugada syndrome. Circ J 2008; 72: 94-101.

9. Kosuge M, Kimura K. Clinical implications of electrocardiograms for patients with non-ST-segment elevation acute coronary syndromes in the interventional era. Circ J 2009; 73: 798-805.

10. Goldberger L. Clinical electrocardiography: A simplified approach. St Louis: Mosby, 1998.

11. Van Leeuwen P, Hailer B, Lange S, Grönemeyer D. Spatial distribution of repolarization times in patients with coronary artery disease. Pacing Clin Electrophysiol 2003; 26: 1706-1714.

12. Hailer B, Chaikovsky I, Auth-Eisernitz S, Schafer H, Van Leeuwen $P$. The value of magnetocardiography in patients with and without relevant stenoses of the coronary arteries using an unshielded system. Pacing Clin Electrophysiol 2005; 28: 8-11.

13. Park JW, Hill PM, Chung N, Hugenholtz PG, Jung F. Magnetocardiography predicts coronary artery disease in patients with acute chest pain. Ann Noninvasive Electrocardiol 2005; 10: 312-323.

14. Tolstrup K, Madsen BE, Ruiz JA, Greenwood SD, Camacho J, Siegel RJ, et al. Non-invasive resting magnetocardiographic imaging for the rapid detection of ischemia in subjects presenting with chest pain. Cardiology 2006; 106: 270-276.

15. Lim HK, Chung NS, Kim K, Ko YG, Kwon H, Lee YH, et al. Can magnetocardiography detect patients with non-ST-segment elevation myocardial infarction? Ann Med 2007; 39: 617-627.

16. Lim HK, Kwon H, Chung NS, Ko YG, Kim JM, Kim IS, et al. Usefulness of magnetocardiogram to detect unstable angina pectoris and non-ST elevation myocardial infarction. Am J Cardiol 2009; 103: $448-454$

17. Takala $\mathrm{P}$, Hänninen $\mathrm{H}$, Montonen J, Korhonen $\mathrm{P}$, Mäkijärvi M, Nenonen J, et al. Heart rate adjustment of magnetic field map rota- tion in detection of myocardial ischemia in exercise magnetocardiography. Basic Res Cardiol 2002; 97: 88-96.

18. Kanzaki H, Nakatani S, Kandori A, Tsukada K, Miyatake K. A new screening method to diagnose coronary artery disease using multichannel magnetocardiogram and simple exercise. Basic Res Cardiol 2003; 98: $124-132$.

19. Kwon H, Kim K, Kim JM, Lee YH, Lim HK, Kim TE, et al. Classification of magnetocardiographic parameters based on probability density function. J Korean Phys Soc 2006; 48: 1114-1116.

20. Lee YH, Kim JM, Kim K, Kwon H, Yu KK, Kim IS, et al. 64channel magnetocardiogram system based on double relaxation oscillation SQUID planar gradiometers. Superconduct Sci Technol 2006; 19: S284-S288.

21. Kim K, Lee YH, Kwon H, Kim JM, Kim IS, Park YK. Averaging algorithm based on data statistics in magnetocardiography. Neurol Clin Neurophysiol 2004; 42.

22. Hämäläinen M. Interpreting magnetic fields of the brain: Minimum norm estimates. Med Biol Eng Comput 1994; 32: 35-42.

23. Engblom H, Foster JE, Martin TN, Groenning B, Pahlm O, Dargie $\mathrm{HJ}$, et al. The relationship between electrical axis by 12-lead electrocardiogram and anatomical axis of the heart by cardiac magnetic resonance in healthy subjects. Am Heart J 2005; 150: 507-512.

24. Braunwald E, Antman EM, Beasley JW, Califf RM, Cheitlin MD, Hochman JS, et al. ACC/AHA guidelines for the management of patients with unstable angina and non-ST-segment elevation myocardial infarction: A Report of the American College of Cardiology/ American Heart Association Task Force on Practice Guidelines (Committee on the management of patients with unstable angina). J Am Coll Cardiol 2000; 36: 970-1062.

25. Fuster V. Acute coronary syndrome: The degree and morphology of coronary stenoses. J Am Coll Cardiol 1999; 34: 1854-1856. 